\title{
Synergistic lubricating effect of graphene/ionic liquid composite material used as an additive
}

\author{
Lincong LIU ${ }^{1}$, Ming ZHOU ${ }^{1, *}$, Youtang MO ${ }^{1}$, Pengpeng BAI ${ }^{2}$, Qilin WEI $^{3}$, Long $\mathrm{JIN}^{1}$, Shengli YOU ${ }^{1}$, Mingyue \\ WANG $^{1}$, Liangchuan LI ${ }^{1}$, Xin CHEN ${ }^{1}$, Xiao LI $^{4}$, Yu TIAN ${ }^{2}$ \\ ${ }^{1}$ School of Mechanical and Transportation Engineering, Guangxi University of Science and Technology, Liuzhou 545006, China \\ ${ }^{2}$ State Key Laboratory of Tribology, Tsinghua University, Beijing 100084, China \\ ${ }^{3}$ Department of Electromechain and Automotive Engineering, Liuzhou City Vocational College, Liuzhou 535006, China \\ ${ }^{4}$ Chengdu Carbon Co., Ltd., Chengdu 610100, China
}

Received: 20 May 2020 / Revised: 28 July 2020 / Accepted: 10 August 2020

(C) The author(s) 2020 .

\begin{abstract}
We prepared a graphene/ionic liquid (G/IL) composite material by the hybridization of $\mathrm{G}$ and an IL for use as a lubricating oil additive. The friction coefficient and wear volume of a base oil containing $0.04 \mathrm{wt} \%$ of the G/IL composite was reduced by $45 \%$ and $90 \%$, respectively. Furthermore, the base oil containing the G/IL composite exhibited better lubricating properties than the base oil containing G, IL, or a mixture of IL and $G$ at the same mass fraction. A synergistic lubrication mechanism was also revealed. The G/IL composite was adsorbed and deposited on the wear surface, forming a more ordered protective film and a unique tribochemical reaction film during rubbing. Therefore, the G/IL composite exhibited the synergistic lubricating effects of $G$ and IL, which significantly improved the lubricating performance of the base oil. This study also suggested a way to limit the out-of-plane puckering of $\mathrm{G}$ at the macroscale.
\end{abstract}

Keywords: grapheme (G); ionic liquid (IL); synergistic lubrication; protective films; out-of-plane puckering mechanism

\section{Introduction}

Excessive wear directly degrades the stability, safety, and reliability of mechanical equipment [1]. The use of lubricants is an effective method of controlling friction and wear in mechanical equipment. Various additives (e.g., zinc dialkyldithiophosphate and metallo-organic compounds) are used to improve the lubricating performance of lubricants to meet the stricter requirements of modern industry. However, these additives contain toxic substances such as sulfur and phosphorus, which can seriously affect the environment. Green additives such as molybdenum disulfide $\left(\mathrm{MoS}_{2}\right)$ and nanocarbon materials are increasingly being developed [2-5].

Graphene $(G)$ is a type of $2 D$ material with a hexagonal honeycomb 2D grid structure; it consists of single-layer carbon atoms exhibiting $\mathrm{sp}^{2}$ hybridization. Monolayer $G$ is currently the thinnest nanomaterial [6]. Owing to its excellent electrical and optical properties, atomic thickness, good mechanical properties, and layered structure with low shear strength, $\mathrm{G}$ can be used in a wide range of applications, such as solar cells, supercapacitors, catalysts, sensors, and lubricants [7-10]. For use in various nano- or micro-scale systems with complex working conditions, $\mathrm{G}$ outperforms traditional lubricating materials [11, 12]. However, it is difficult to disperse $\mathrm{G}$ stably in a lubricating medium because of its chemical inertness, which significantly affects its tribological performance. In 2001, Ye et al. [13] used an ionic liquid (IL) as a lubricant for various

*Corresponding author: Ming ZHOU, E-mail: zhoum03@163.com 
friction pairs for the first time and revealed its excellent antifriction performance and broad application prospects. The use of ILs as oil- or water-based lubricants has been studied [14]. ILs have a wider application range than traditional solvents owing to their thermal stability, chemical stability, non-flammability, and inherent polarity [15]. More recently, the functional combination of an IL and $\mathrm{G}$ has become important for the development of hybrid nanomaterials with synergistic characteristics for use as lubricating additives [16, 17]. Zhou et al. [18] dispersed $G$ in a polymerized imidazole IL through noncovalent $\pi-\pi$ interaction and suggested that hybrid G/IL additives have excellent potential for application. More research has shown that $G$ functionalized by an IL may be highly suitable for application as a lubricating additive [19-22].

The out-of-plane puckering mechanism of $\mathrm{G}$ has been studied experimentally on the nanoscale using atomic force microscopy tips and $G$ sheets [23-25]. Li et al. [26] revealed that the friction of 2D materials such as $G$ increases monotonously with decreasing number of layers on a weakly bonded substrate owing to the elastic deformation and puckering of the material. Smolyanitsky et al. [27] performed a Brownian dynamics simulation and found that the relaxation and surface compliance of samples affected the friction of few-layer G. Zheng et al. $[28,29]$ revealed the energy barrier and friction source of $G$ in both commensurate and incommensurate friction at the atomic scale. These studies showed that the adsorption and wrinkle deformation of $G$ at the interface has an important effect on its surface friction reduction performance. In addition, the studies have shown that the surface energy of the IL, which is close to that of G, can effectively reduce the stacking effect caused by van der Waals forces. On the basis of these studies, we proposed that the IL may limit the out-of-plane puckering effect, which could enhance the lubricating properties of G. We prepared a G/IL composite material by liquid-phase ultrasound-assisted methods and investigated its microstructural characteristics and tribological performance as an additive.

\section{Experimental section}

\subsection{Material preparation}

The G used in this study was produced by Tsinglube Technologies Co., Ltd. (Liuzhou). The IL (purity: 99\%) was purchased from the Lanzhou Institute of Chemical Physics, Chinese Academy of Sciences (Lanzhou). Its main physical and chemical properties are shown in Table 1. PAO6 base oil was purchased from Beijing Sunright Trade Co., Ltd. (Beijing). Petroleum ether and absolute ethanol were purchased for use as analytical reagents from Chengdu Chron Chemicals Co., Ltd. (Chengdu) and used as received.

\subsection{Experimental procedure}

The G/IL composite material was prepared by adding $G$ and IL to absolute ethanol at a mass ratio of 3:1. The mixture was stirred uniformly by a magnetic stirrer (Changzhou) for $1 \mathrm{~h}$ at 1,200 rpm and room temperature. Then the mixture was ultrasonically dispersed for $3 \mathrm{~h}$ in an ultrasonic cleaner (Skymen Cleaning Equipment Shenzhen Co., Ltd., Shenzhen). The product, a somewhat viscous black G/IL composite, was obtained using a vacuum drying oven (Wuxi Marit Technology Co., Ltd., Wuxi, China) and dried at $70{ }^{\circ} \mathrm{C}$ for further use.

Scanning electron microscopy (SEM, Quanta 200 FEG, FEI, the Netherlands) equipped with energy dispersive spectrometer (EDS) was used to characterize the morphology and microstructure of

Table 1 Main physical and chemical properties of IL used in this study.

\begin{tabular}{|c|c|c|c|c|c|}
\hline IL & \multicolumn{5}{|c|}{ Basic information } \\
\hline N-butyl & Chemical formula & $\begin{array}{l}\text { Molecular } \\
\text { formula }\end{array}$ & Density $\left(\mathrm{g} / \mathrm{cm}^{3}\right)$ & Viscosity (cp) & $\begin{array}{l}\text { Decomposition } \\
\text { temperature }\left({ }^{\circ} \mathrm{C}\right)\end{array}$ \\
\hline tetrafluoroborate & & $\mathrm{C}_{9} \mathrm{H}_{14} \mathrm{BF}_{4} \mathrm{~N}$ & 1.21 & 160 & 342 \\
\hline
\end{tabular}


the samples. The interlayer distance was characterized by X-ray diffraction (XRD, Rigaku, Japan) using $\mathrm{Cu} K \alpha$ radiation in a $2 \theta$ range of $5^{\circ}-60^{\circ}$. The characteristic peaks of the nanoparticles were obtained in a range of $1,000-3,000 \mathrm{~cm}^{-1}$ by Raman spectroscopy (Raman, Horiba JobinYvon, France) using $532 \mathrm{~nm}$ laser excitation. The elemental compositions and ratios of the nanoparticles were determined by X-ray photoelectron spectroscopy (XPS, PHI Quantera II, Ulvac-Phi Inc., Japan) using Al $\mathrm{K} \alpha$ radiation and a C 1s binding energy of $284.8 \mathrm{eV}$.

The tribological properties of pure PAO6 and PAO6 containing G, the IL, a mixture of $G$ and the IL $(\mathrm{IL}+\mathrm{G})$, or the $\mathrm{G} / \mathrm{IL}$ composite were measured using an optimal SRV-4 high-temperature tribotester. A steel ball (diameter: $10.3 \mathrm{~mm}, \mathrm{GCr} 15)$ was pressed onto a fixed steel disc ( $\phi 24 \mathrm{~mm} \times 7.88 \mathrm{~mm}, \mathrm{GCr} 15)$ to obtain steel/steel point contact. After $0.1 \mathrm{~mL}$ of a lubricant was applied to the ball-disc contact area, the steel ball was driven in a friction test with a stroke of $1 \mathrm{~mm}$ (load: $25 \mathrm{~N}$, frequency: $25 \mathrm{~Hz}$ ) for $20 \mathrm{~min}$. The steel disc was processed uniformly by mechanical polishing to eliminate the effects of surface roughness. The steel balls and steel disc were ultrasonically cleaned with petroleum ether and absolute ethanol before the friction experiments [30]. All the experiments were performed at a temperature of $25 \pm 1{ }^{\circ} \mathrm{C}$ and relative humidity of $60 \%-70 \%$. At least two tribological tests were performed to eliminate the effects of accidental errors, and the average values were obtained. The steel disc was cleaned after each test using petroleum ether and ethanol in sequence until the remaining lubricant on the surface was removed to avoid interference with the experimental results. A 3D white-light interferometer (Nexview, ZYGO, Lambda, USA) was used to investigate the morphological features and wear volume of the wear surfaces on the steel disc [31]. The rubbing surfaces and the protective film formed on the wear scars were observed using Raman spectroscopy and XPS.

\section{Results and discussion}

\subsection{Characterization of G/IL composite material}

SEM is a powerful tool for characterizing the morphology and structure of carbon materials. SEM images of the $G$ and G/IL composite are shown in Figs. 1(a) and 1(b), respectively. The G/IL composite has a layered structure similar to that of G. Both materials have a relatively complete graphite-like structure with obvious stacking. In addition, Figs. 1(c) and $1(d)$ showed that the $G$ contained only $C$ and $O$, whereas the G/IL composite contained not only $\mathrm{C}$ and $\mathrm{O}$ but also $\mathrm{F}$.

The microstructures of the G and G/IL composite were characterized using Raman spectroscopy, XRD,
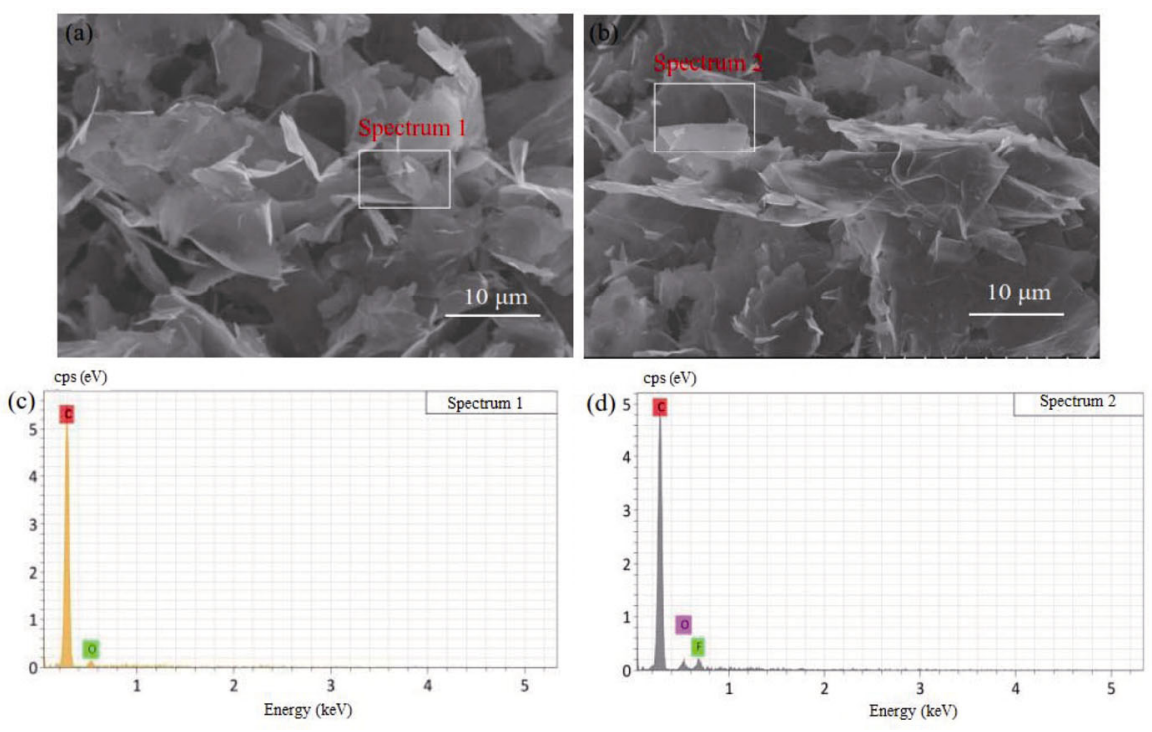

Fig. 1 SEM images of (a) G and (b) G/IL composite and EDS analysis of (c) G and (d) G/IL composite. 

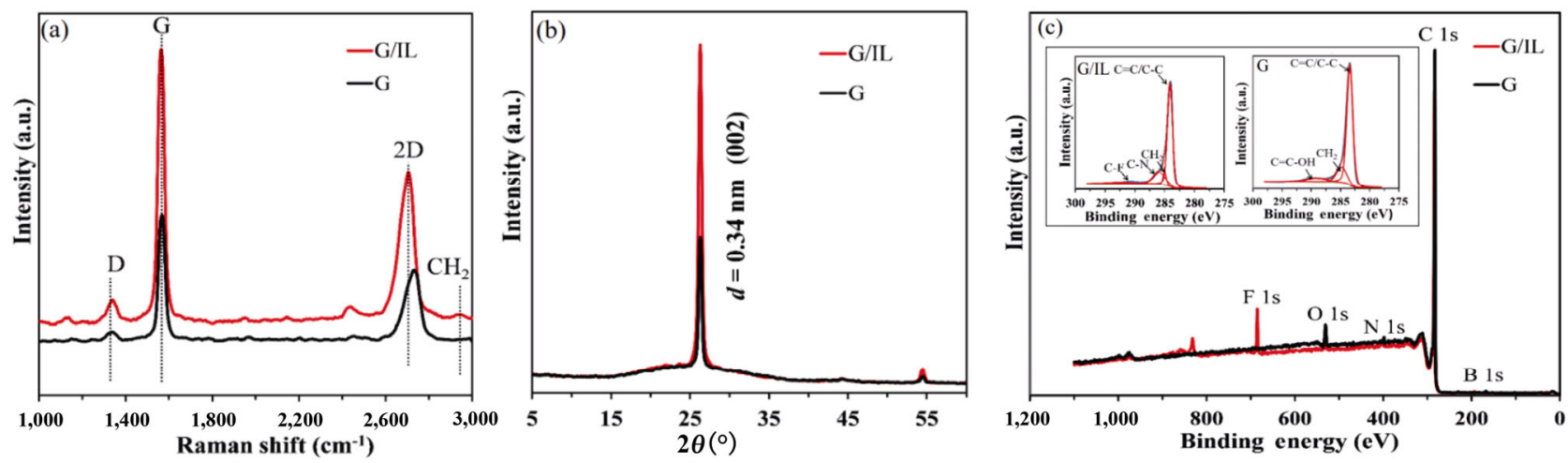

Fig. 2 (a) Raman spectra, (b) XRD patterns, and (c) full and C 1s XPS spectra of G and G/IL composite.

and XPS. Figure 2(a) shows the Raman spectra of the samples. The G/IL composite and G had clear D, G, and 2D peaks at approximately 1,350, 1,580, and $2,700 \mathrm{~cm}^{-1}$, respectively. The $\mathrm{D}$ peak indicates the presence of defects and disorder in carbonbased materials. The $G$ peak results from the Brillouin/optical zone center vibrations with $E_{2 g}$ symmetry, which are associated with the regular $\mathrm{sp}^{2}$ carbon skeleton. The $\mathrm{G}$ peak is often used to measure the degree of crystallization of graphitebased materials. The position of the $2 \mathrm{D}$ peak and the intensity ratio of the $G$ peak to the $2 D$ peak $\left(I_{\mathrm{G}} / I_{2 \mathrm{D}}\right)$ indicate the number of layers in carbonbased materials [32]. A single layer is present when $I_{\mathrm{G}} / I_{2 \mathrm{D}}<0.7$, and a double layer is present when $I_{\mathrm{G}} / I_{2 \mathrm{D}}$ is between 0.7 and 1.0. When $I_{\mathrm{G}} / I_{2 \mathrm{D}}>$ 1.0, a multilayer is generated [33]. The G peak is significantly more intense than the $2 \mathrm{D}$ peak, indicating that both materials are multilayered. The intensity ratio of the $D$ peak to the $G$ peak $\left(I_{\mathrm{D}} / I_{\mathrm{G}}\right)$ can be used to evaluate the defect density of carbon-based materials. The $I_{\mathrm{D}} / I_{\mathrm{G}}$ ratios of the G/IL composite and G were 0.189 and 0.268 , respectively, indicating that the $G$ had slightly fewer defects after hybridization. The G/IL composite exhibited clear diffraction peaks at a wavelength of approximately $2950 \mathrm{~cm}^{-1}$, which can be attributed to the methylene $\mathrm{CH}_{2}$ of the pyridine ring and carbon chain of the IL. This result indicated that the IL was successfully grafted onto the G surface.

As shown in Fig. 2(b), strong (002) diffraction peaks appeares only at $2 \theta=26^{\circ}$ in the XRD patterns of the G and G/IL composite. According to the Bragg equation [34]:

$$
2 d \sin \theta=n \lambda
$$

where $n=1, \lambda=1.54184 \AA(1 \AA=0.1 \mathrm{~nm})$, and the corresponding interlayer distance $d$ is $0.34 \mathrm{~nm}$. No other strong diffraction peaks were detected, indicating that oxygen-containing functional groups were not introduced into either material by functionalization.

Figure 2(c) shows the full spectra and C 1s spectra of the G/IL composite and G. Peak fitting of the XPS spectra was performed using a Gaussian-Lorentzian function after Shirley background correction was applied [32]. The C 1 s spectrum of $G$ was fitted to three Gaussian peaks at binding energies of 283.4, 284.7, and $289.1 \mathrm{eV}$, which correspond to the carbon skeleton $(\mathrm{C}=\mathrm{C} / \mathrm{C}-\mathrm{C})$, methylene $\left(\mathrm{CH}_{2}\right)$, and carboxyl $(\mathrm{O}=\mathrm{C}-\mathrm{OH})$, respectively. The $\mathrm{C} 1 \mathrm{~s}$ spectrum of the G/IL composite was fitted to four Gaussian peaks at $284.1,285,285.8$, and $292.5 \mathrm{eV}$, which correspond to $\mathrm{C}=\mathrm{C} / \mathrm{C}-\mathrm{C}, \mathrm{CH}_{2}, \mathrm{C}-\mathrm{N}$, and $\mathrm{C}-\mathrm{F}$, respectively [35]. This result indicates the successful preparation of the G/IL composite.

\subsection{Dispersion of G/IL composite material}

The dispersion stability of the G/IL composite and $I L+G$ mixture in the PAO6 base oil was observed (Fig. 3). After $6 \mathrm{~h}$, the two samples had a similar appearance, and no obvious precipitation had occurred. After $12 \mathrm{~h}$, the bottom of the PAO6 base oil mixed with $G$ and the IL began to settle, and the central part was transparent, whereas the $\mathrm{G} / \mathrm{IL}$ composite was well-dispersed. After $24 \mathrm{~h}$, the sample containing the $\mathrm{IL}+\mathrm{G}$ mixture had almost completely precipitated, whereas the PAO6 base oil containing the G/IL composite exhibited good dispersion stability without settling. 
(a) $12 \mathrm{~h}$

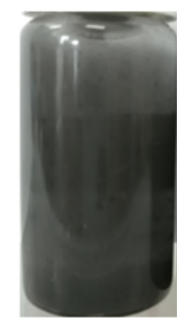

(b)

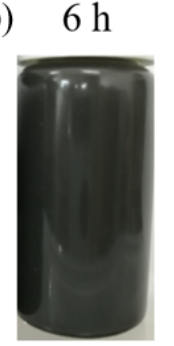

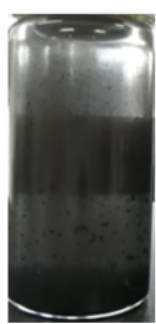

$12 \mathrm{~h}$

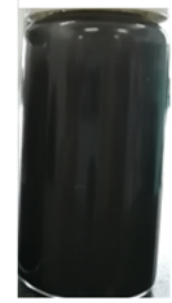

$24 \mathrm{~h}$

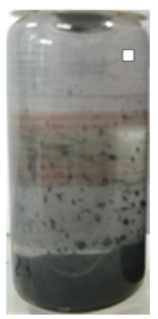

$24 \mathrm{~h}$

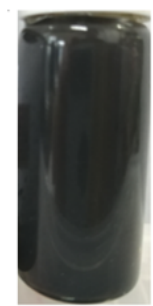

Fig. 3 Dispersion stability of (a) IL + G mixture and (b) G/IL composite in PAO6 base oil.

\subsection{Tribological properties of G/IL composite material}

We investigated the tribological properties of the G/IL composite at various concentrations in the PAO6 base oil. Figure 4 shows the friction coefficient curves and the average friction coefficient of the G/IL composite in the base oil. The friction coefficient of pure PAO6 was very high, with a maximum value of approximately 0.33 , and the friction coefficient fluctuated considerably. However, using the G/IL composite as an additive greatly improved the tribological performance of the base oil. The friction coefficient was greatly reduced, and the friction coefficient curves became smoother and more stable. We observed that the friction coefficient initially decreased and then increased with increasing concentration of the G/IL composite. The lowest friction coefficient was obtained at a concentration of $0.04 \mathrm{wt} \%$.

We evaluated the lubricating performance of each additive at a concentration of $0.04 \mathrm{wt} \%$ to confirm the superior performance of the G/IL composite. Figure 5 shows the friction coefficient, average friction coefficient, wear width and wear volume of pure PAO6 and PAO6 containing $0.04 \mathrm{wt} \%$ of the IL+G mixture, G, the IL, or the G/IL composite. The introduction of these four additives significantly affected the lubricating properties of PAO6. The friction coefficient curves of the base oil containing

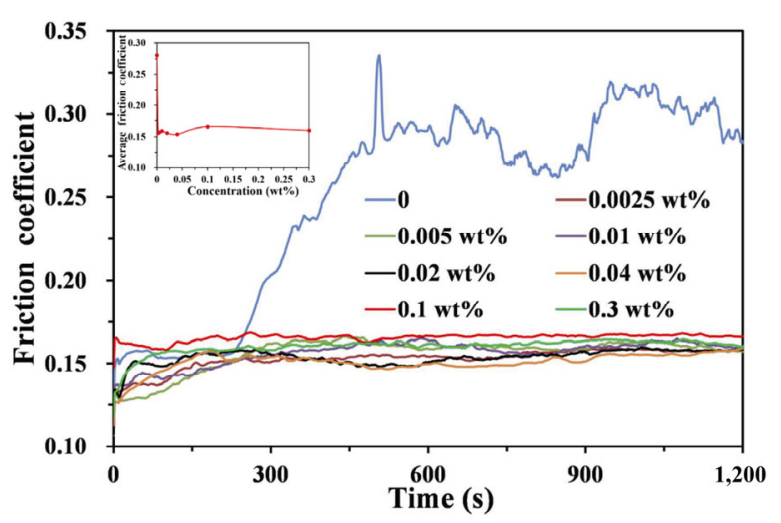

Fig. 4 Friction coefficient curves and average friction coefficient (inset) of base oil containing G/IL composite at various concentrations.

each additive became smooth and stable, and the friction coefficient and wear volume were effectively reduced. Specifically, the average friction coefficient and wear volume of the base oil containing the G/IL composite were reduced by $45 \%$ and $91 \%$, respectively, compared with those of PAO6. Furthermore, we observed that the base oil containing the G/IL composite had better lubricating properties than the base oil containing $G$, the IL, or the IL+G mixture at the same mass fraction. This result proved that the introduction of the G/IL composite substantially improved the lubricating properties of the PAO6 base oil.

The 3D morphologies and 2D wear profiles of the wear surface of the steel discs lubricated with pure PAO6 and PAO6 containing $0.04 \mathrm{wt} \%$ of the IL+G mixture, G, the IL, and the G/IL composite were investigated; the results are shown in Fig. 6. The surface of the steel disc lubricated with pure PAO6 had the most severe wear and contained many deep rough scratches. Therefore, PAO6 exhibited the lowest lubricating performance, which resulted in the most severe wear. The observations also indicated that abrasive wear occurred on the wear surfaces. The wear width and depth on the steel discs lubricated with the base oil containing $G$, the $\mathrm{IL}$, and the IL+G mixture were smaller, and the defects became shallower and narrower. Note that the wear surface of the steel disc lubricated with base oil containing the G/IL composite had the smallest wear width and depth; besides, its wear surface had almost no scratches and was very 

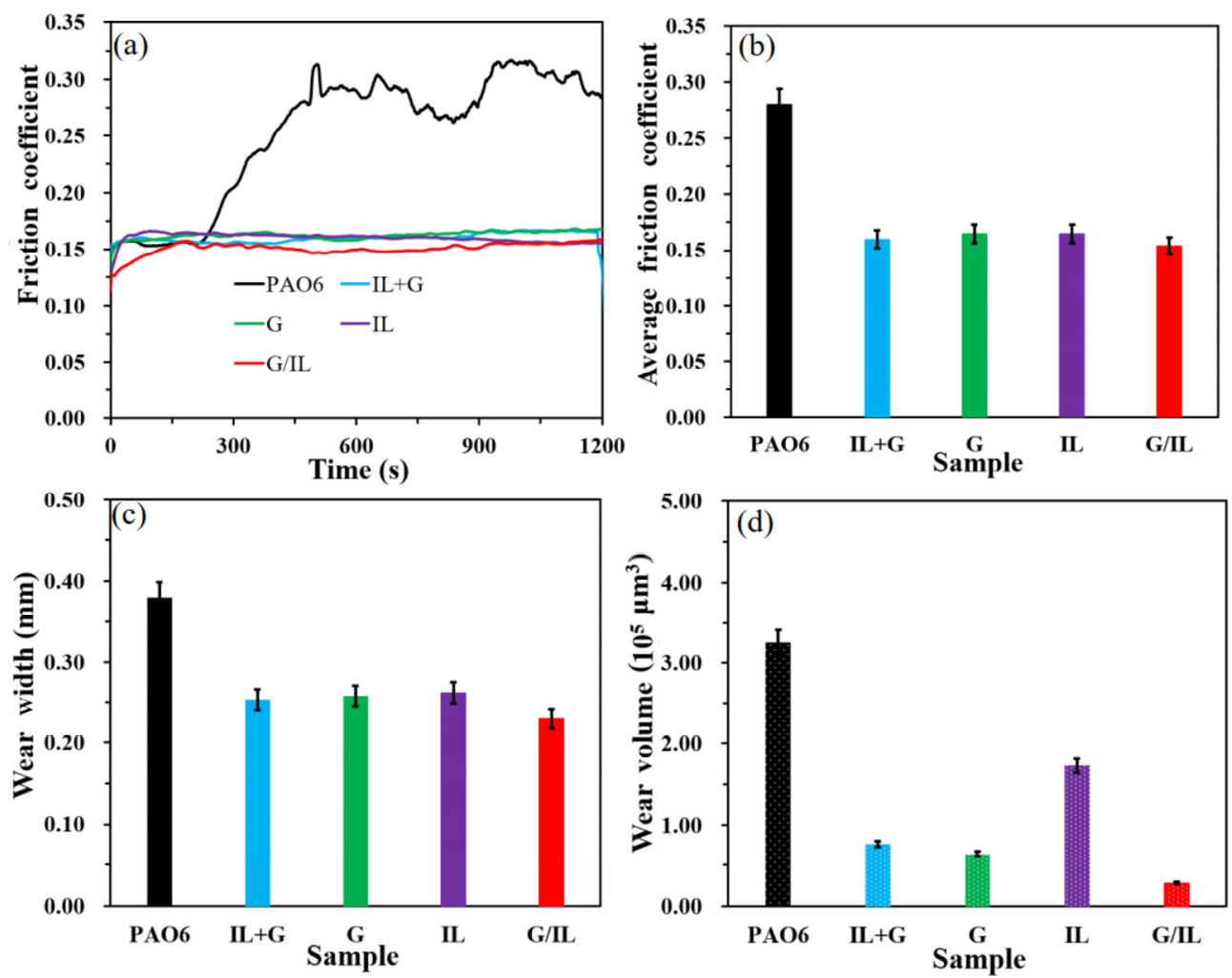

Fig. 5 (a) Friction coefficient curves, (b) average friction coefficient, (c) wear width, and (d) wear volume of pure PAO6 and PAO6 cont aining $0.04 \mathrm{wt} \%$ of IL $+\mathrm{G}$ mixture, G, IL, or G/IL composite.
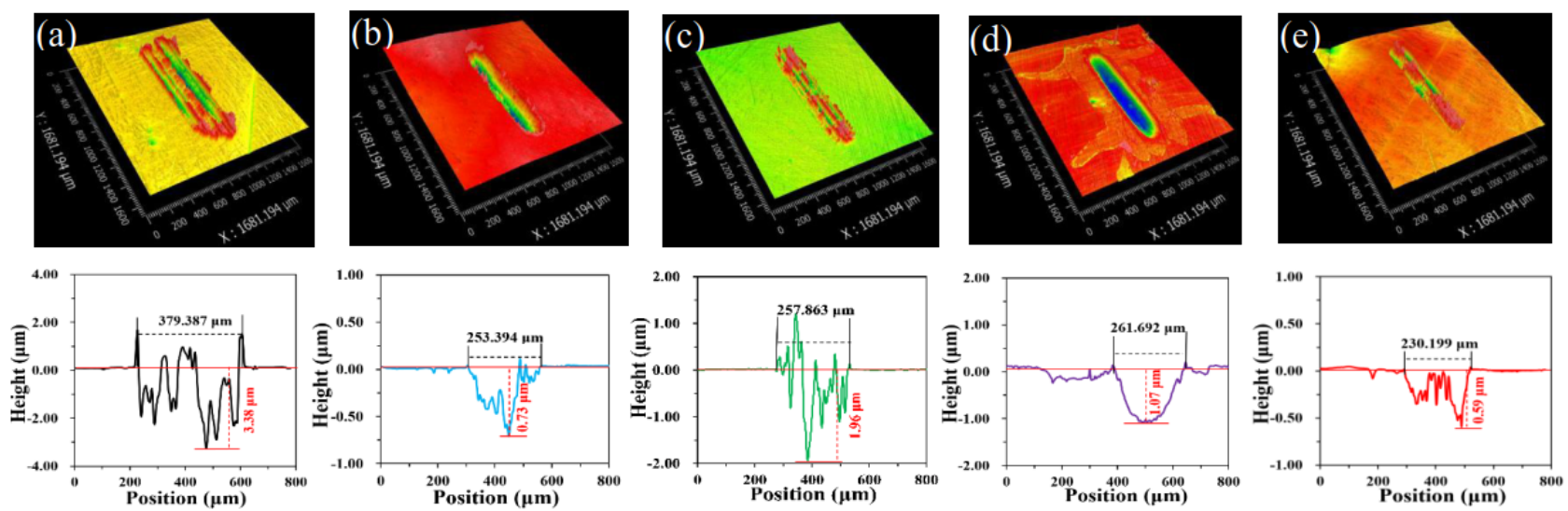

Fig. 6 Morphology of wear surface of steel disc lubricated with (a) pure PAO6 and PAO6 containing 0.04 wt $\%$ of (b) IL $+\mathrm{G}$ mixture, (c) G, (d) IL, or (e) G/IL composite.

smooth. Therefore, the G/IL composite exhibited the best antiwear performance among the additives. In summary, the base oil containing the G/IL composite had better lubricating properties than pure PAO6 and the base oil containing $G$, the $\mathrm{IL}$, or the $\mathrm{IL}+\mathrm{G}$ mixture. This result also demonstrated that the G/IL composite was a nanocomposite material with excellent lubricating ability.

We used a four-ball tester to investigate the tribological properties of pure PAO6 and PAO6 containing the IL+G mixture, $G$, the IL, and the G/IL composite $(0.04 \mathrm{wt} \%)$ at room temperature and demonstrated the advantages of using the G/IL composite as a base oil additive. Figure 7 shows the friction coefficient curves, average friction coefficients, and wear diameters of the five samples. As in the SRV tribological experiments, the G/IL composite had the lowest average friction 

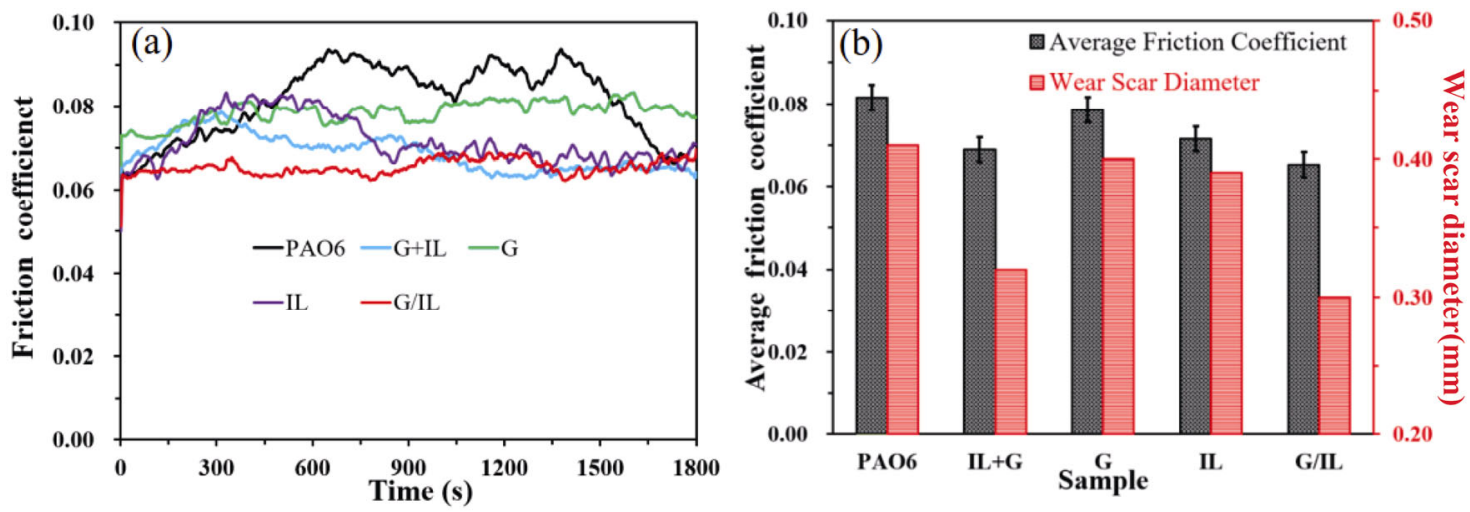

Fig. 7 (a) Friction coefficient curves and (b) average friction coefficient and wear diameter of pure PAO6 and PAO6 containing $0.04 \mathrm{wt} \%$ of $\mathrm{IL}+\mathrm{G}, \mathrm{G}, \mathrm{IL}$, or G/IL composite obtained using a four-ball tester.

coefficient and wear diameter, with a fairly smooth friction coefficient curve, indicating excellent antifriction performance. In addition, the worn area of the steel ball lubricated with PAO6 alone and PAO6 containing the G/IL composite were observed using SEM; the results are shown in Figs. 8(a) and 8(b), respectively. The wear scar of the steel ball lubricated with PAO6 is large, and the worn surface is very rough. The steel ball lubricated with PAO6 and the G/IL composite clearly has a much narrower wear scar, and the worn surface is very smooth. Therefore, the G/IL composite has excellent potential for use as a base oil additive in antifriction and antiwear applications.

\subsection{Synergistic lubrication mechanism of G/IL composite material}

Figure 9 shows the Raman spectra of the wear surfaces of steel discs lubricated with pure PAO6 and PAO6 containing the IL+G mixture, G, the IL, or the G/IL composite at a concentration of $0.04 \mathrm{wt} \%$. The wear surfaces of the steel discs lubricated with all five samples showed a strong diffraction peak near $2,850 \mathrm{~cm}^{-1}$, which was tentatively attributed to $\mathrm{CH}_{2}$ methylene and may represent the protective oil film on the friction surface resulting from rubbing with the base oil. In addition, the wear surfaces of the steel discs lubricated with all five samples also showed a strong diffraction peak near $640 \mathrm{~cm}^{-1}$, which represented iron oxide. This result indicated that a tribochemical reaction occurred during rubbing [36]. When the wear surface of the steel disc was lubricated with the base oil containing $G$ or the $\mathrm{IL}+\mathrm{G}$ mixture, $\mathrm{D}$ peaks near $1,350 \mathrm{~cm}^{-1}$ were observed. It can be concluded that protective films containing carbonaceous materials appeared on the wear track, which explained the decreases in the friction coefficient and wear volume. However, we observed that no $G$ peak appeared near $1,580 \mathrm{~cm}^{-1}$

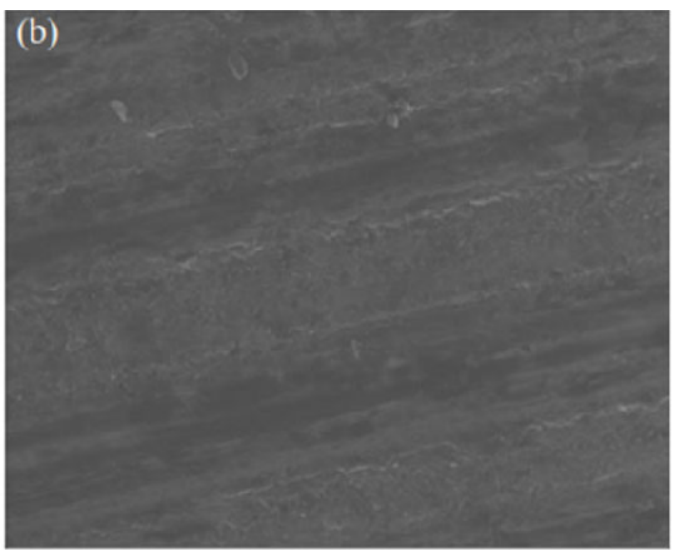

Fig. 8 SEM images of worn area of steel ball lubricated with (a) PAO6 and (b) PAO6 containing 0.04 wt $\%$ of G/IL composite. 


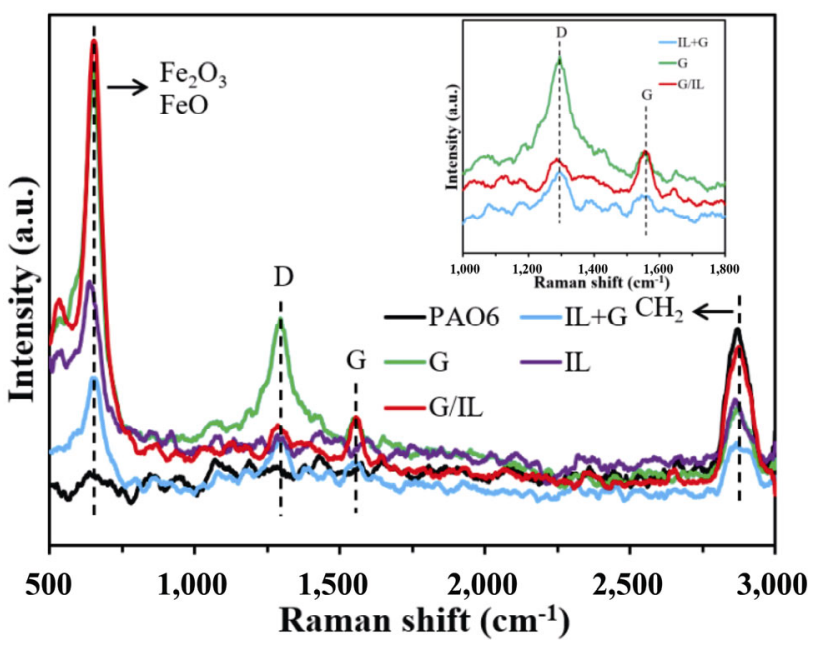

Fig. 9 Raman spectra of wear surface of steel discs lubricated with pure PAO6 and PAO6 containing $0.04 \mathrm{wt} \%$ of IL $+\mathrm{G}, \mathrm{IL}, \mathrm{G}$, and G/IL composite.

for the wear surfaces of the steel discs lubricated with the base oil containing $G$ or the IL+G mixture, indicating that the protective films were severely damaged under the high contact pressure. The wear surface of the steel disc lubricated with the base oil containing the IL did not show clear D and $G$ peaks, indicating that the surface did not adsorb carbonaceous materials during rubbing. The wear surface of the steel disc lubricated with the base oil containing the G/IL composite showed the characteristic $D$ and $G$ peaks, indicating that a wellordered carbonaceous protective film was formed and stably adsorbed on the wear track. This film prevented direct contact with the friction surface and reduced friction and wear. We attributed the formation of an ordered carbonaceous protective film on the surface of the steel disc lubricated with the base oil containing the G/IL composite to the hybridization of the IL and $G$ by the $\pi-\pi$ interaction. The IL can regulatethe adsorption effect of $G$ and suppress the wrinkling of $G$ during rubbing, which significantly improved the stability of the adsorption film.

Figure 10 shows the XPS full spectra and typical element spectra of the wear surfaces of steel discs lubricated with pure PAO6 and PAO6 containing the IL+G mixture, G, the IL, or the G/IL composite at a concentration of $0.04 \mathrm{wt} \%$. B and $\mathrm{F}$ did not appear in the wear tracks of the steel discs lubricated with pure PAO6 and the base oil containing G, which indicated that a severe fluorination reaction did not occur. The wear tracks of the steel discs lubricated with the base oil containing the IL and G/IL composite had high concentrations of B, F, and $\mathrm{Fe}$, indicating that the friction chemical reaction produced fluoride. A chemical reaction film containing fluoride was deposited and absorbed on the contact interfaces and reduced friction and wear. Although $\mathrm{B}$ and $\mathrm{F}$ also appeared in the wear track of the steel disc lubricated with the base oil containing the IL $+G$ mixture, a high concentration of $C$ and low concentrations of $\mathrm{O}$ and $\mathrm{Fe}$ were also observed. These results showed that the friction surface was covered with a relatively thick carbonaceous film resulting from the agglomeration of G. The friction chemical reactions on the surface may have been suppressed; however, severe dry friction occurred between the carbon films. This greatly damaged the carbon film and caused severe surface wear. The $\mathrm{C} 1 \mathrm{~s}$ peak at $284.8 \mathrm{eV}$, the $\mathrm{O} 1 \mathrm{~s}$ peak at approximately $532 \mathrm{eV}$, and the $\mathrm{N}$ 1s peak at $399.8 \mathrm{eV}$ in the spectra of the wear track of the steel disc lubricated with the base oil containing the G/IL composite (Figs. 10(b), 10(c), and 10(f), respectively) indicate the formation of a carbonaceous film and nitrogen oxides. In addition, Figs. 10(d) and 10(e) show a strong Fe $2 \mathrm{p}$ peak at $711.1 \mathrm{eV}$ and a strong $\mathrm{F}$ 1s peak at $686.5 \mathrm{eV}$, indicating that complex oxides such as $\mathrm{Fe}_{2} \mathrm{O}_{3}, \mathrm{Fe}_{3} \mathrm{O}_{4}$, or $\mathrm{FeF}_{2}$ are present on the wear surface [37]. The tribological chemical reaction that may occur on the surface of a worn steel block is

$$
\mathrm{Fe}^{2+}+2 \mathrm{~F}^{-}=\mathrm{FeF}_{2} ; 2 \mathrm{Fe}^{3+}+3 \mathrm{O}^{2-}=\mathrm{Fe}_{2} \mathrm{O}_{3}
$$

The lubrication mechanism of $G$ and an IL has been thoroughly discussed. Tang et al. [38] prepared a graphene oxide (GO)/IL material by amidation and cation $-\pi$ stacking for use as an additive and revealed synergistic lubrication mechanisms. The IL functional groups of the GO/IL material are absorbed on the rubbing surface owing to electrostatic interactions, which promote the formation of a boundary tribofilm consisting of a GO/IL deposition film and a chemical reaction film. Therefore, it can be concluded that the G/IL composite is adsorbed and deposited on the wear surface and forms a carbonbased protective film. In addition, it can generate a 


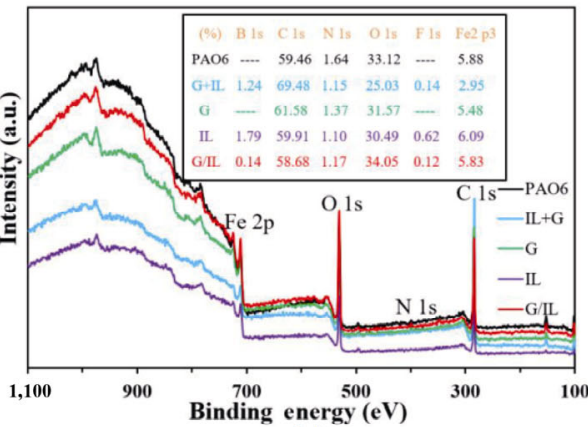

(a)

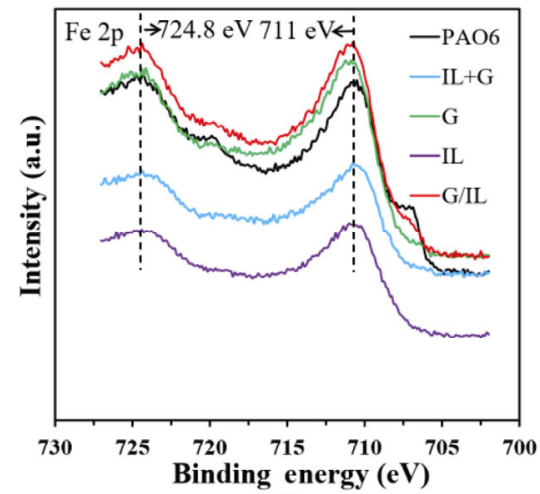

(d)

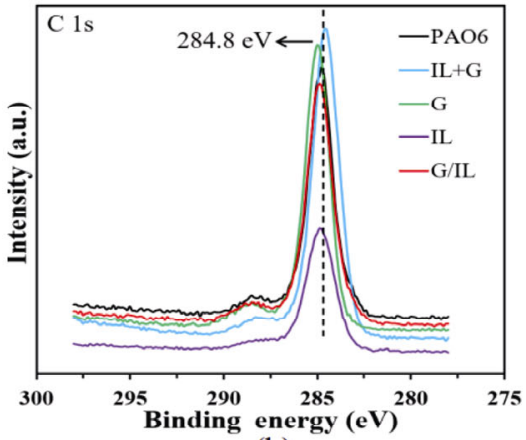

(b)

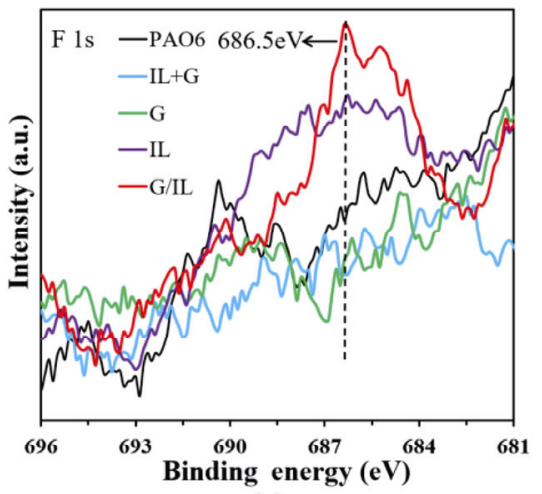

(e)

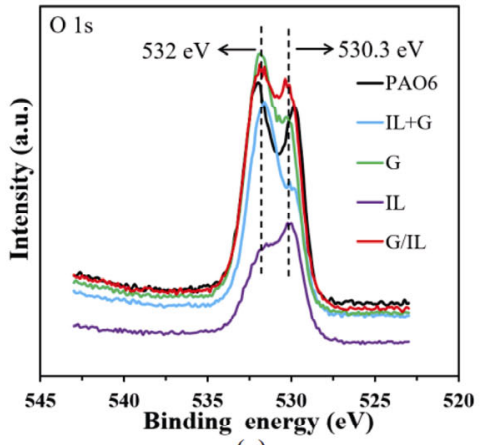

(c)

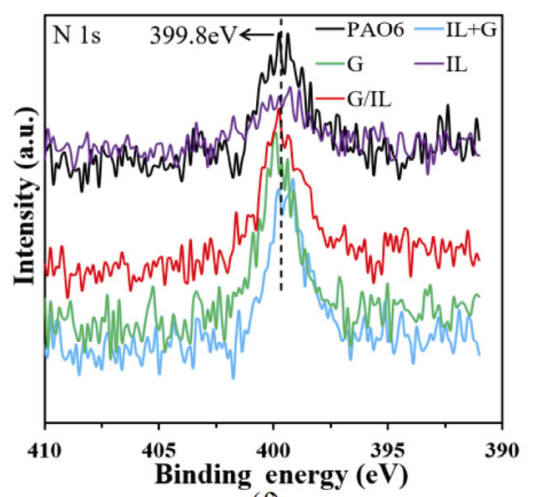

(f)

Fig. 10 XPS spectra of wear surfaces of steel discs lubricated with pure PAO6 and PAO6 containing 0.04 wt $\%$ of the IL $+\mathrm{G}$ mixture, the IL, G, or the G/IL composite: (a) full spectra, (b) C 1s spectra, (c) O 1s spectra, (d) Fe 2p spectra, (e) F 1s spectra, and (f) N 1s spectra.

tribochemical reaction at the steel interface to form a tribochemical reaction film that protects the contact surface. This result also demonstrates that the remarkable lubricating performance of the G/IL composite is related to the synergistic lubricating effect of $G$ and the IL.

To illustrate the lubricating effect of the G/IL composite, Figure 11 shows a schematic of the lubrication mechanism during the friction process when the G/IL composite is used as an additive in the PAO6 base oil. On the basis of previous understanding, we speculated that pinning of $G$ at the friction interface by the IL can suppress the wrinkling of $G$ during rubbing owing to the $\pi-\pi$ interaction, which substantially improved the stability of the adsorption membrane and the lubricating effect of $\mathrm{G}$. In addition, there is a strong F 1s peak at the wear scar of the steel disc lubricated

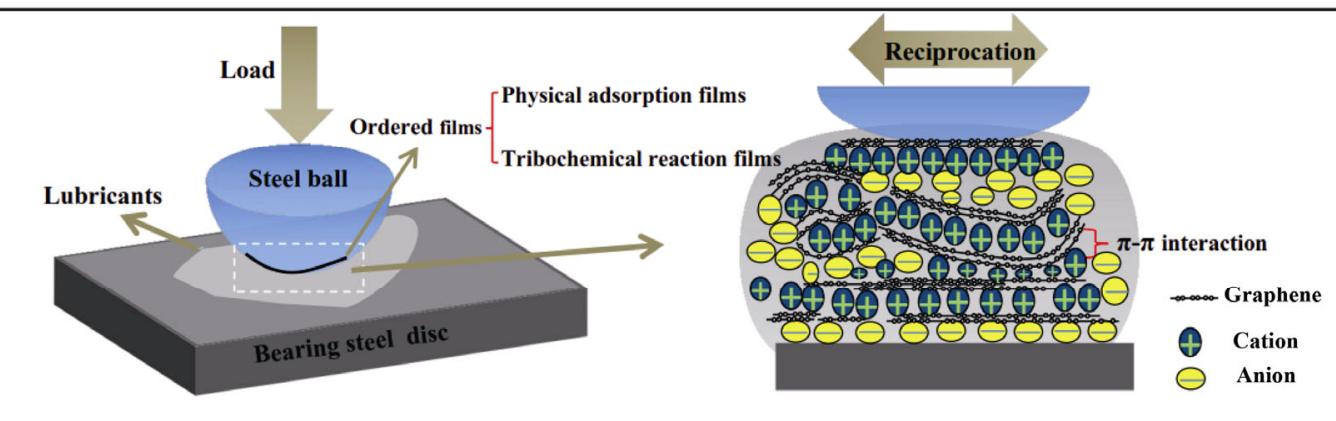

Fig. 11 Schematic of lubrication mechanism of G/IL composite used as additive in PAO6 base oil during rubbing. 
with the base oil containing the G/IL composite, indicating that an appropriate $\mathrm{G}$ content also promotes the complex tribochemical reaction that forms protective tribochemical reaction films containing compounds such as $\mathrm{Fe}_{2} \mathrm{O}_{3}$ and $\mathrm{FeF}_{2}$ and significantly enhances the lubricating effect of the IL. Therefore, the G/IL composite exhibited excellent synergistic lubrication by the IL and G and substantially reduced friction and wear on the contact surfaces.

\section{Conclusions}

A G/IL composite material was successfully prepared using liquid-phase ultrasound-assisted methods. At a concentration of $0.04 \mathrm{wt} \%$, the G/IL composite considerably improved the lubricating properties of base oil and substantially decreased friction and wear on the contact surfaces. Specifically, the friction coefficient and wear volume of the base oil with $0.04 \mathrm{wt} \%$ of the G/IL composite were reduced by $45 \%$ and $90 \%$, respectively. Furthermore, the lubricating properties of the G/IL composite were also superior to those of $G$, the IL, or an IL+G mixture at the same mass fraction. We characterized the wear surface of the steel disc and concluded that the pinning of $G$ at the friction interface by the IL can suppress the wrinkling of $G$ during rubbing owing to the $\pi-\pi$ interaction. Thus, G is adsorbed and deposited on the wear surface to form a more ordered protective film. The presence of an appropriate amount of $\mathrm{G}$ also promoted the tribochemical reaction of the IL at the friction interface to produce a tribochemical reaction film. Therefore, the G/IL composite exhibits the synergistic lubricating effect of $G$ and the IL and thus significantly improves the lubricating performance of the PAO6 base oil. These results suggest that the developed G/IL composite has potential applications as an additive in various com posite materials, including lubricants. They also suggest that it is important to investigate the synergistic lubrication mechanism of other lubricating additives for both scientific and industrial applications.

\section{Acknowledgements}

This work was supported by the National Natural
Science Foundation of China (Grant No. 12062002), Guangxi Natural Science Foundation (Grant No. 2018GXNSFAA138174), and the Science and Technology Development Plan of Liuzhou (No. 2018CB10508).

Open Access This article is licensed under a Creative Commons Attribution 4.0 International License, which permits use, sharing, adaptation, distribution and reproduction in any medium or format, as long as you give appropriate credit to the original author(s) and the source, provide a link to the Creative Commons licence, and indicate if changes were made.

The images or other third party material in this article are included in the article's Creative Commons licence, unless indicated otherwise in a credit line to the material. If material is not included in the article's Creative Commons licence and your intended use is not permitted by statutory regulation or exceeds the permitted use, you will need to obtain permission directly from the copyright holder.

To view a copy of this licence, visit http:// creativecommons.org/licenses/by/4.0/.

\section{References}

[1] Holmberg K, Andersson P, Erdemir A. Global energy consumption due to friction in passenger cars. Tribol Int 47: 221-234 (2012)

[2] Wang W, Xie G X, Luo J B. Black phosphorus as a new lubricant. Friction 6(1): 116-142 (2018)

[3] Rengifo S, Zhang C, Harimkar S, Boesl B, Agarwal A. Effect of $\mathrm{WS}_{2}$ addition on tribological behavior of aluminum at room and elevated temperatures. Tribol Lett 65(3): 76 (2017)

[4] Lv Y, Wang W, Xie G X, Luo J B. Self-lubricating PTFE-based composites with black phosphorus Nanosheets. Tribol Lett 66(2): 61 (2018)

[5] Xu Y F, Peng Y B, Dearn K D, Zheng X J, Yao L L, Hu $X$ G. Synergistic lubricating behaviors of graphene and $\mathrm{MoS}_{2}$ dispersed in esterified bio-oil for steel/steel contact. Wear 342-343: 297-309 (2015)

[6] Berman D, Erdemir A, Sumant A V. Graphene: A new emerging lubricant. Mater Today 17(1): 31-42 (2014)

[7] Chen Z F, Wang Z, Li X M, Lin Y X, Luo N Q, Long M $\mathrm{Z}$, Zhao $\mathrm{N}$, $\mathrm{Xu} \mathrm{J}$ B. Flexible piezoelectric-induced pressure sensors for static measurements based on nanowires/Graphene Heterostructures. ACS Nano 11(5): 
4507-4513 (2017)

[8] Li X M, Lv Z, Zhu H W. Carbon/silicon Heterojunction solar cells: State of the art and prospects. Adv Mater 27(42): 6549-6574 (2015)

[9] Liu L C, Zhou M, Li X, Jin L, Su G S, Mo Y T, Li L C, Zhu H W, Tian Y. Research progress in application of 2D materials in liquid-phase lubrication system. Materials 11(8): 1314 (2018)

[10] Zhang W, Zhou M, Zhu H W, Tian Y, Wang K L, Wei J Q, Ji F, Li X, Li Z, Zhang P, et al. Tribological properties of oleic acid-modified graphene as lubricant oil additives. J Phys D Appl Phys 44(20): 205303 (2011)

[11] Liu L C, Zhou M, Jin L, Li L C, Mo Y T, Su G S, Li X, Zhu $\mathrm{H} \mathrm{W}$, Tian Y. Recent advances in friction and lubrication of graphene and other 2D materials: Mechanisms and applications. Friction 7(3): 199-216 (2019)

[12] Xiao H P, Liu S H. 2D nanomaterials as lubricant additive: A review. Mater Design 135: 319-332 (2017)

[13] Ye C F, Liu W M, Chen Y X, Yu L G. Room-temperature ionic liquids: A novel versatile lubricant. Chem Commun 21:2244-2245 (2001)

[14] Wang Y R, Yu Q L, Cai M R, Shi L, Zhou F, Liu W M. Ibuprofen-based ionic liquids as additives for enhancing the lubricity and Antiwear of water-ethylene glycol liquid. Tribol Lett 65(2): 55 (2017)

[15] Gusain R, Gupta P, Saran S, Khatri O P. Halogen-free bis(imidazolium)/bis(ammonium)-di[bis(salicylato)bora te] ionic liquids as energy-efficient and environmentally friendly lubricant additives. ACS Appl Mater Interfaces 6(17): 15318-15328 (2014)

[16] Kinoshita H, Kondo M, Nishina Y, Fujii M. Anti-wear effect of Graphene oxide in lubrication by fluorinecontaining ionic liquid for steel. Tribol Online 10(1): 91-95 (2015)

[17] Khare V, Pham M Q, Kumari N, Yoon H S, Kim C S, Park J I, Ahn S H. Graphene-ionic liquid based hybrid Nanomaterials as novel lubricant for low friction and wear. ACS Appl Mater Interfaces 5(10): 4063-4075 (2013)

[18] Zhou X S, Wu T B, Ding K L, Hu B J, Hou M Q, Han B $\mathrm{X}$. Dispersion of graphene sheets in ionic liquid [bmim] $\left[\mathrm{PF}_{6}\right]$ stabilized by an ionic liquid polymer. Chem Commun 46(3): 386-388 (2010)

[19] Parviz D, Irin F, Shah S, Das S, Sweeney C B, Green M J. Challenges in liquid-phase exfoliation, processing, and assembly of pristine Graphene. Adv Mater 28(40): 8796-8818 (2016)

[20] Wang X Q, Fulvio P F, Baker G A, Veith G M, Unocic R R, Mahurin S M, Chi M F, Dai S. Direct exfoliation of natural graphite into micrometre size few layers graphene sheets using ionic liquids. Chem Commun
46(25): 4487-4489 (2010)

[21] Liang Y Y, Wu D Q, Feng X L, Müllen K. Dispersion of Graphene sheets in organic solvent supported by ionic interactions. Adv Mater 21(17): 1679-1683 (2009)

[22] Elbourne A, McLean B, Voïtchovsky K, Warr G G, Atkin R. Molecular resolution in situ imaging of spontaneous Graphene exfoliation. J Phys Chem Lett 7(16): 3118-3122 (2016)

[23] Cho D H, Wang L, Kim J S, Lee G H, Kim E S, Lee S, Lee S Y, Hone J, Lee C. Effect of surface morphology on friction of graphene on various substrates. Nanoscale 5(7): 3063-3069 (2013)

[24] Lee C, Li Q Y, Kalb W, Liu X Z, Berger H, Carpick R W, Hone J. Frictional characteristics of atomically thin sheets. Science 328(5974): 76-80 (2010)

[25] Liu S W, Wang H P, Xu Q, Ma T B, Yu G, Zhang C H, Geng D C, Yu Z W, Zhang S G, Wang W Z, et al. Robust microscale superlubricity under high contact pressure enabled by graphene-coated microsphere. Nat Commun 8(1): 14029 (2017)

[26] Li Q Y, Lee C, Carpick R W, Hone J. Substrate effect on thickness-dependent friction on graphene. Phys Status Solidi B 247(11-12): 2909-2914 (2010)

[27] Smolyanitsky A, Killgore J P, Tewary V K. Effect of elastic deformation on frictional properties of few-layer graphene. Phys Rev B 85: 035412 (2012)

[28] Zheng X H, Gao L, Yao Q Z, Li Q Y, Zhang M, Xie X M, Qiao S, Wang G, Ma T B, Di Z F, et al. Robust ultra-low-friction state of graphene via moiré superlattice confinement. Nat Commun 7(1): 13204 (2016)

[29] Xu Q, Li X, Zhang J, Hu Y Z, Wang H, Ma T B. Suppressing nanoscale wear by graphene/graphene interfacial contact architecture: A molecular dynamics study. ACS Appl Mater Interfaces 9(46): 40959-40968 (2017)

[30] Zhao J, He Y Y, Wang Y F, Wang W, Yan L, Luo J B. An investigation on the tribological properties of multilayer graphene and $\mathrm{MoS}_{2}$ nanosheets as additives used in hydraulic applications. Tribol Int 97: 14-20 (2016)

[31] Zhao J, Li Y R, Wang Y F, Mao J Y, He Y Y, Luo J B. Mild thermal reduction of graphene oxide as a lubrication additive for friction and wear reduction. $R S C$ Adv 7(3): 1766-1770 (2017)

[32] Gusain R, Mungse H P, Kumar N, Ravindran T R, Pandian R, Sugimura H, Khatri O P. Covalently attached graphene-ionic liquid hybrid nanomaterials: Synthesis, characterization and tribological application. J Mater Chem A 4(3): 926-937 (2016)

[33] Peng L Q, Xie J H, Guo C, Zhang D. Review of characterization methods of graphene. J Funct Mater 44(21): 3055-3059 (2013) 
[34] He T X, Dai Q W, Huang W, Wang X L. Colloidal suspension of graphene oxide in ionic liquid as lubricant. Appl Phys A 124(11): 777 (2018)

[35] Fan X Q, Wang L P. High-performance lubricant additives based on modified graphene oxide by ionic liquids. J Colloid Interface Sci 452: 98-108 (2015)

[36] Chourpa I, Douziech-Eyrolles L, Ngaboni-Okassa L, Fouquenet J F, Cohen-Jonathan S, Soucé M, Marchais H, Dubois P. Molecular composition of iron oxide nanoparticles, precursors for magnetic drug targeting, as

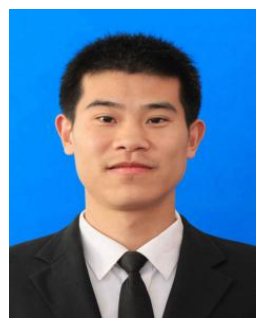

Lincong LIU. He received his B.S. degree in mechatronic engineering in 2017 from Harbin University of Science and Technology and his

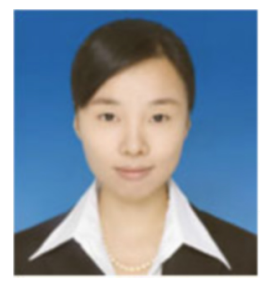

Ming ZHOU. She is a professor and a master supervisor at School of Mechanical Engineering, Guangxi University of Science and Technology. She graduated from Tsinghua University in 2007 and obtained her Ph.D. degree in 2013 from State Key laboratory of Tribology, Tsinghua University. She is invited to be a lecturer of the "Applied Technology Talents Training Project of Made in China 2025 Plan” by the Ministry of Industry and Information Technology. Her research interest is characterized by confocal Raman microspectroscopy. Analyst 130(10): 1395-1403 (2005)

[37] Zheng D, Wu Y P, Li Z Y, Cai Z B. Tribological properties of $\mathrm{WS}_{2}$ /graphene nanocomposites as lubricating oil additives. RSC Adv 7(23): 14060-14068 (2017)

[38] Tang W W, Huang Y Z, Wang B G. Synthesis of ionic liquid functionalized graphene oxides and their tribological property under water lubrication. Fuller Nanotub Car N 26(3): 175-183 (2018)

M.S. degree from Guangxi University of Science and Technology in 2020. His research interests include liquid-phase lubrication and micro-tribology.

the regulation and application of mechanical interface behavior based on new materials. She has published over 21 papers in well-known international and domestic journals and 16 papers are included in SCI cited over 300 times. She has received the Outstanding Award for Excellent Mechanical Engineering Doctoral Thesis of the Society of Mechanical Engineering of China, the first prize of the outstanding doctoral thesis of Tsinghua University, and the promising academic young person of China Academy of Engineering Physics. She is also selected as the "BaGui area Young Scholars of Guangxi". 